\title{
Author Index to Vol. 31, 1963
}

A

Adachi, M.

Ado, K.

Ando, T.

Aoki, K.

Asada, K.

Ashimura, S.

Atarashiya, K.

F

Fujii, Y.

Fujimura, Z.

Funaki, K.

Furumi, S.

G

Gotoda, M.

H

Hayami, H.

Hayami, F.

Hisamatsu, Y.

Hisano, S.

Hisano, $\mathrm{T}$

I

Ichikawa, E.

Imaoka, Y.

Inagaki, M.

Ino, $\mathrm{H}$.

Inoue, $\mathrm{M}$.

Ishibashi, N.

Ishida, $\mathrm{T}$.

Ishii, Y.

Ishikawa, $\mathrm{T}$.

Ishino, $\mathrm{T}$.

Ito, $\mathrm{K}$.

$\mathbf{K}$

Kainuma, K.

Kamiya, S.

Kato, O.

Kisaki, $\mathrm{H}$.
62

65,74

Page

196

70,196

70

63

74

150

74

153

7,191

67

66,160

62

70

188,189

178

67

78

64

$64,65,66,74,75$

76

$154,168,188$

187

152,192

187

$64,65,66,75$

77

188

73

75

195
Kondo, Y.

54,77

Kotera, Y.

34,174

Koyama, Y.

187

Kozawa, A.

$71,72,151$

Kozuka, Z.

77

Kubo, T.

160

Kuroda, T.

$68,75,153$

150

Kusaba, I.

M

Maezawa, H. 198

Makino, S.

75

Masuko, N.

189

Matsumoto, O.

$68,75,153$

Matsushima, T.

152

Mine, $\mathrm{T}$.

62

Mizutani, Y.

67

Momoki, K.

192

Motomura, $\mathrm{H}$.

67

Mukaibo, T.

79

N

Nagamatsuya, T. $\quad 76,195$

Namikawa, M. $\quad 64$

Naraoka, K. 174

Narasimhan, K.R. 42

Nobuoka, S. $\quad 70,196$

Noda, H. $\quad 77$

Nomura, S. $\quad 54,77$

O

Odo, K. $\quad 78$

Okada, S. $\quad 63$

Okamoto, H. 71

Okaniwa, H. 194

Okinaka, Y. 194

Omori, G. $\quad 76,152$

Onoue, Y. 67

Osafune, H. 24,78 
S

Saeki, Y.

Saito, T.

Sakai, W.

Sakashita, H.

Sato, E.

Sato, M.

Sawada, S.

Seiyama, T.

Seki, K.

Sekido, S.

Sekine, T.

Sekino, J.

Shenoi, B.A.

Shiokawa, J.

Shiota, K.

Shirai, K.

Shiratori, $\mathrm{H}$.

Sugimura, K.

Sugino, K.

Suzuki, S.

\section{T}

\section{Takagi, M.}

Takahashi, T.

Takatsuki, M.

Takei, T.

Takemura, N.

Takeshima, $\mathrm{T}$.
76,152

79

1,198

160

153

$64,69,153$

$153,189,190$

187

74

15,192

34,78

192

42

77

77

78

206

62

78

152,194

76

$64,152,188$

74

155

193

79
Tamura, $\mathrm{H}$.

Tanaka, S.

Tochihara, S.

Toshima, S.

Tsuji, F.

Tsunoi, M.

U

Uchimura, K. $\quad 67$

Ueda, S.

63

Ueda, Y.

W

Watanabe, A. $\quad 63$

Watanabe, N.

$\mathbf{Y}$

Yamada, T.

69

Yamane, R.

67,68

Yamamoto, O.

64,152

153,189

76,195

47,78

$64,65,66,74,75$

76

15,192

$72,73,190,191$

34

$63,153,154,168,187,188$

24,78

64

63

189

Yasufuku, M.

Yokoh, T.

Yokota, Y.

Yonemura, M.

Yoshizawa, S. 